ARTICLE

\title{
Structural basis of ribosomal RNA transcription regulation
}

\author{
Yeonoh Shin (1) 1,3, M. Zuhaib Qayyum,3, Danil Pupov (1) 2, Daria Esyunina², Andrey Kulbachinskiy (1) ${ }^{2}$ \& \\ Katsuhiko S. Murakami (iD ${ }^{1 \otimes}$
}

Ribosomal RNA ( $r R N A$ ) is most highly expressed in rapidly growing bacteria and is drastically downregulated under stress conditions by the global transcriptional regulator DksA and the alarmone ppGpp. Here, we determined cryo-electron microscopy structures of the Escherichia coli RNA polymerase (RNAP) $\sigma^{70}$ holoenzyme during rRNA promoter recognition with and without DksA/ppGpp. RNAP contacts the UP element using dimerized $\alpha$ subunit carboxylterminal domains and scrunches the template DNA with the $\sigma$ finger and $\beta^{\prime}$ lid to select the transcription start site favorable for rapid promoter escape. Promoter binding induces conformational change of $\sigma$ domain 2 that opens a gate for DNA loading and ejects $\sigma_{1.1}$ from the RNAP cleft to facilitate open complex formation. DksA/ppGpp binding also opens the DNA loading gate, which is not coupled to $\sigma_{1.1}$ ejection and impedes open complex formation. These results provide a molecular basis for the exceptionally active rRNA transcription and its vulnerability to $\mathrm{DksA} / \mathrm{ppGpp}$. 
D acteria sense the availability of nutrition and adjust ribosome biogenesis to optimize their growth. The rate of ribosome biogenesis is primarily determined by rRNA transcription $^{1,2}$, which constitutes as much as $70 \%$ of total RNA synthesis and is initiated approximately every second from each of the seven rRNA operons ( $r r n \mathrm{~A}-\mathrm{E}$ and $r r n \mathrm{G}-\mathrm{H})$ in E. coli during exponential growth ${ }^{3}$. However, it is drastically repressed under stress conditions such as nutrient-starved stationary phase ${ }^{4}$. rRNA expression is primarily regulated at the initiation stage of RNA synthesis, including RNAP binding to promoter DNA, unwinding the DNA, and escaping from the promoter.

The promoters (e.g., $r r n B P 1$ ) for expressing rRNA operons are unique compared with other $\sigma^{70}$-dependent promoters, including (1) the A+T-rich UP element located upstream of the -35 element (from -60 to -40 ); (2) the $\mathrm{G}+\mathrm{C}$-rich discriminator sequence downstream of the -10 element (from -8 to -1 ); and (3) the transcription start site (TSS) located nine bases downstream from the -10 element (Fig. 1a and Supplementary Fig. 1a). The UP element is recognized by the carboxyl-terminal domain of the a subunit (aCTD) and enhances rRNA transcription by more than 30 -fold ${ }^{5}$. The $\mathrm{G}+\mathrm{C}$-rich discriminator and unusual TSS selection of rRNA promoters make its open complex (RPo) unstable, but facilitate RNAP escape from the promoter by reducing abortive RNA cycle prior to the RNA elongation stage ${ }^{6}$. These promoter elements play key roles in the wide range of rRNA transcription regulation between nutrientrich and -poor growth conditions.

rRNA transcription activity is regulated by two small moleculesthe initiating ribonucleotide (iNTP) (ATP in the case of $r r n B 1)^{7}$ and the bacterial alarmone ppGpp (guanosine tetraphosphate, aka "magic spot"), which is an allosteric effector of the RNAP-binding global transcription regulator $\mathrm{Dks}^{8-10}$. In the presence of high iNTP concentration, rRNA synthesis starts immediately after RNAP formed the RPo on rRNA promoters, allowing rapid transition to transcription elongation (promoter escape). However, the iNTPlimited condition shifts the equilibrium to favor early intermediates in promoter complex formation, including the closed complex, which is further shifted by DksA/ppGpp binding to RNAP $^{4}$. The ppGpp concentration is increased under stress conditions, which enhances DksA-mediated rRNA repression by stabilizing RNAP-DksA complex in a functionally important binding mode ${ }^{11}$.

The majority of bacterial RNAP-DNA complex structures determined by X-ray crystallography contain short promoter DNA fragments with a premelted transcription bubble that mimics RPo to maximize its stability required for timeconsuming crystallization method ${ }^{12,13}$. These studies explained the structural basis of promoter recognition and transcript initiation but left unexplored the interactions of RNAP with duplex DNA around the UP element (via aCTDs) and the
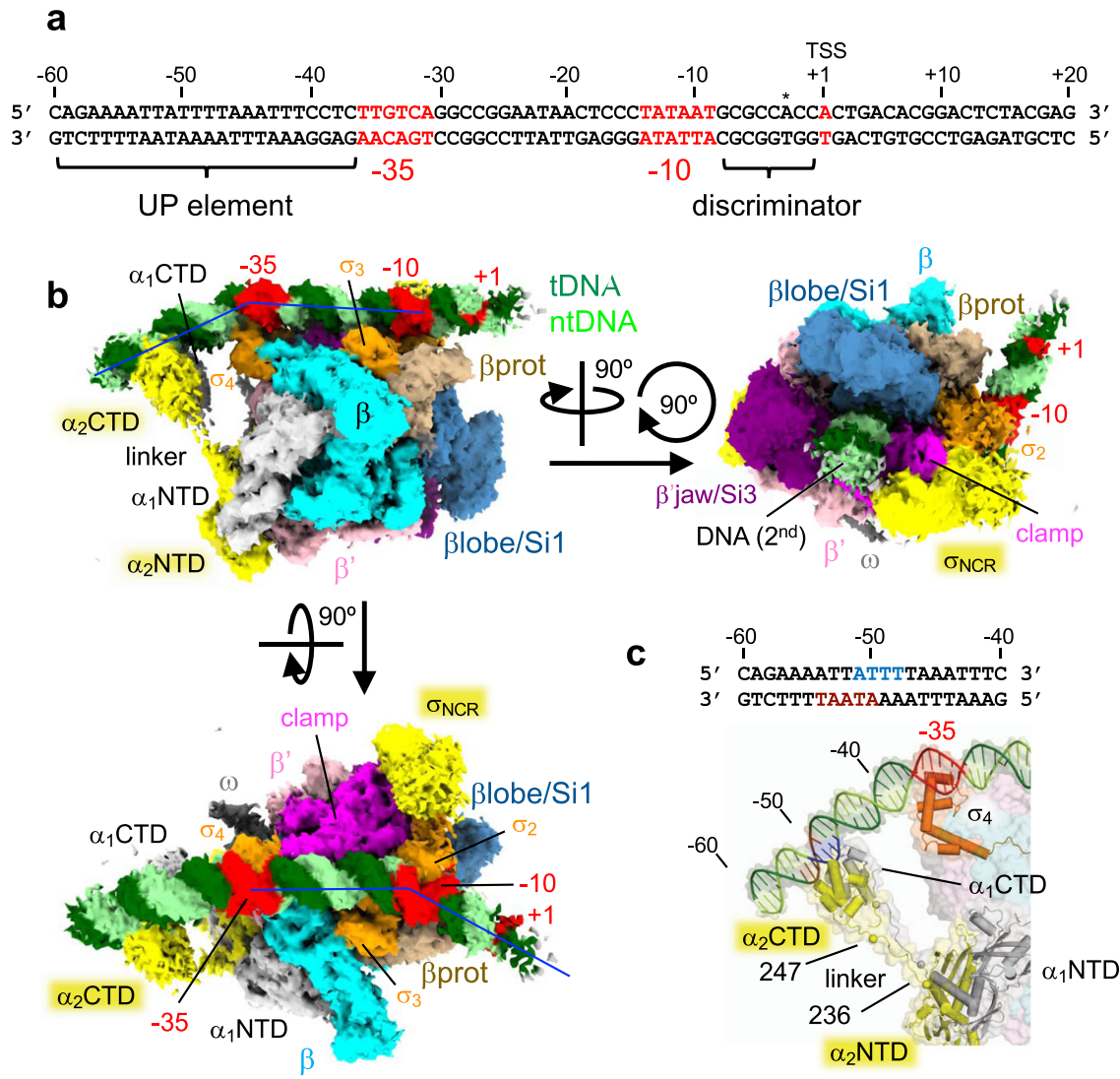

Fig. 1 Cryo-EM structure of the RNAP-rrnBP1 closed complex (RPC). a The sequence of the E. coli rrnBP1 promoter DNA used for cryo-EM. The UP element, -35 element, -10 element, transcription start site $(T S S,+1)$ and discriminator sequence are indicated. Alternative TSS from the nonscrunched open complex is indicated by an asterisk. $\mathbf{b}$ Orthogonal views of the RPc cryo-EM density map. Subunits and domains of RNAP and DNA are colored and labeled ( $\beta$ prot, $\beta$ protrusion; tDNA, template DNA; ntDNA, nontemplate DNA). The density of downstream DNA beyond the +4 position is not traceable. Blue lines denote the direction of the DNA axis, with kinks at -37 and -13. The second DNA at the RNAP cleft is indicated (DNA (2nd)). c A magnified view showing the $\alpha$ CTDs and UP element interaction. The domains of $\alpha$ subunits, $\sigma_{4}$, and DNA are depicted as ribbon models with a partially transparent surface. At the top, the sequence of the UP element is shown. The ntDNA ( -51 to -48 ) and tDNA ( -54 to -50 ) sequences binding $\alpha_{1}$ CTD and $\alpha_{2}$ CTD are highlighted in blue and brown, respectively. 
contacts with the -10 element (via $\sigma$ domain 2, residues 92-127 and 373-456 in $\sigma^{70}$ ) in a closed complex and the scrunched DNA bubble in the RPo formed with the rRNA promoters.

Cryo-electron microscopy (cryo-EM) structures of the E. coli RNAP-rpsTP2 promoter complex with a ppGpp-insensitive DksA homolog TraR revealed the RPo formation pathway in the presence of $\mathrm{TraR}^{14}$. However, the rpsTP2 promoter for expressing ribosomal protein $\mathrm{S} 20$ is distinct from the $\mathrm{rrnBP} 1$ promoter that it contains $\mathrm{G}+\mathrm{C}$-rich DNA upstream of the -35 element and the TSS 7 bases downstream from the -10 element; therefore, it does not reveal the pathway for rRNA promoter complex formation and the mechanism of rRNA transcription regulation. In addition, the presence of TraR does not allow to infer the unperturbed pathway of the open complex formation by RNAP $^{15-17}$. Here, we used cryo-EM to visualize the RNAP and rrnBP1 complexes and two additional complexes with DksA/ ppGpp on the way to RPo formation.

\section{Results}

Cryo-EM structure of the RNAP and rrnBP1 promoter closed complex. To obtain promoter complexes of RNAP with $r r n P 1$, we preincubated $E$. coli RNAP $\sigma^{70}$ holoenzyme with promoter DNA (Fig. 1a) at $37^{\circ} \mathrm{C}$ for $5 \mathrm{~min}$; as a possible way to stabilize the complex, we also added NTPs (ATP and the nonhydrolyzable CTP analog CMPCPP for +1 and +2 NTPs, respectively) prior to cryo-EM grid preparation. In the course of cryo-EM data processing, 3D classification revealed two distinct structures ("Methods" and Supplementary Fig. 2), corresponding to a closed promoter complex (here designated $\mathrm{RPc}$ ) and the transcript initiation complex (RPtic) containing 2-mer RNA. In the RPtic, the 2-mer RNA transcript (5'-CpA-3') base-pairs with $-1 \mathrm{G}$ and $+1 \mathrm{~T}$ in template DNA and is positioned in the posttranslocated conformation. The detailed RPtic structure will be described in a separate report.

We determined the RNAP-rrnBP1 RPc structure with an overall resolution of $4.14 \AA$ (Supplementary Table 1 ). The cryoEM density shows that RNAP binds the duplex DNA from -60 to +3 , which remains fully double-stranded (Fig. 1b, Supplementary Fig. 4 and Supplementary Movie 1), but the density of downstream DNA beyond position +4 is not traceable. Instead, a second DNA binds to the RNAP cleft due to ejection of $\sigma_{1.1}$ from the RNAP cleft during RPc formation as described later.

The cryo-EM density for both aCTDs (residues 248-329), the linkers (residues 236-247) connecting to aNTDs (residues 1-235), and the UP element DNA were traceable in the RPc, allowing us to investigate how each aCTD binds to the UP element unambiguously (Fig. 1c and Supplementary Movie 1). Two aCTDs form a head-to-tail dimer and bind DNA side-byside in the middle of the UP element $(-51$ to -48 on nontemplate DNA (ntDNA) and -54 to -50 on template DNA (tDNA)), which is in good agreement with the DNA footprinting results ${ }^{18}$. Although a subunits form a homodimer, two a subunits play different roles in RNAP, with one $\left(\alpha_{1}\right)$ adjacent to the $\beta$ subunit and the other $\left(\alpha_{2}\right)$ adjacent to the $\beta^{\prime}$. Compared to the $\alpha_{2} \mathrm{CTD}$, the $\alpha_{1}$ CTD is positioned proximally to the -35 element, which explains the result of DNA cleavage by hydroxyl radicals from chelated $\mathrm{Fe}$ attached at each of the two aCTDs $^{19}$. The aCTDs bind DNA with a narrow minor groove, which is formed due to the presence of an $\mathrm{A} / \mathrm{T}$ stretch sequence, as revealed by the recent X-ray crystallographic study of the aCTD and UP element interaction ${ }^{20}$. The side chains of R265 and N294 from both aCTDs are inserted into the DNA minor groove, and basic residues (K291 and K298) are involved in salt bridges with the DNA phosphate backbone (Supplementary Fig. 5a). The linkers of both a subunits are fully extended, and slight DNA bending centered at the -37 position is required for the aCTDs binding to the UP element (Fig. 1c). Consistent with this observation, shortening of the linkers by only three amino acids reduces $r r n B P 1$ transcription ${ }^{21}$. Several studies have proposed that distant upstream DNA (near the -100 position) warps around RNAP on the RPo formation pathway, and the interaction of aCTDs and the UP element is one of the major driving forces for this DNA wrapping ${ }^{22,23}$. However, aCTDs do not bend the DNA around their binding site in the RPc structure determined in this study. This observation suggests that the contacts of aCTDs with the UP element by themselves are not sufficient for wrapping of upstream DNA around RNAP. This may possibly result from the destabilization of upstream DNA and RNAP interactions by truncation of the promoter fragment at the -60 position and/or from specific conditions used for the cryo-EM sample preparation.

The position and orientation of aCTDs in the $r r n B P 1 \mathrm{RPc}$ structure are distinct from those of aCTDs in the RNAP complex with the rpsTP2 promoter, lacking the UP element (PDB: 6PSQ) ${ }^{14}$, which binds DNA just upstream of the $\sigma$ domain $4\left(\sigma_{4}\right)$ bound at the -35 element (Supplementary Fig. 5b). This indicates that the mode of aCTDs interactions with upstream DNA can be significantly different in various promoters depending on the presence of the UP element.

The RPc structure shows how $\sigma_{2}$ binds the duplex form of the -10 element. The DNA encoding the -10 element is anchored by $\sigma$ domain 2 and slightly bends around the upstream edge of the -10 element, allowing the downstream part beyond the -10 element to reach the other side of the RNAP cleft comprising the $\beta$ protrusion domain (Fig. $1 \mathrm{~b}$ and Supplementary Movie 1$)$. The $\sigma$ region $2.3\left(\sigma_{2.3}\right.$, residues $417-434$ ) contacts the -10 element by fitting into the DNA major groove seemingly without sequence-specific interaction, indicating that $\sigma_{2.3}$ recognizes the shape and/or curvature around the -10 element. This finding is in agreement with the previous proposal $^{24}$ that $\sigma^{70}$ does not contact the -10 element DNA bases when it is in duplex form.

Cryo-EM structure of the RNAP and rrnBP1 promoter open complex (RPo). To obtain the structure of the open RNAP-rrnBP1 complex (RPo) with melted DNA bubble, we preincubated RNAP holoenzyme and promoter DNA at $37^{\circ} \mathrm{C}$ for $5 \mathrm{~min}$ prior to cryo-EM grid preparation (Supplementary Fig. 3). 3D classification revealed one major class of particles corresponding to RPo, and its structure was determined with an overall resolution of $3.5 \AA$. In comparison with the RPc, the RPo structure shows significant differences in the UP element (from -60 to -40 ), the downstream DNA (from -14 to +20$)$ and the conformation of the $\sigma$ factor. The cryo-EM density for RPo covers DNA from -44 to +20 , including an open bubble from -13 to +2 and the downstream DNA accommodated in the RNAP cleft (Fig. 2a, Supplementary Fig. 4b, e, and Supplementary Movie 2). In contrast to the RPc, aCTDs and UP elements are disordered.

Basic residues in the $\beta$ lobe (K163, K169, K191, R202, and K203), $\beta$ 'jaw (K1151, K1167, K1170, K1172, and R1174) and $\beta$ 'clamp (R133, K213, K216, K219, and R311) participate in the interaction with downstream DNA to stabilize the RPo (Fig. 2b). The importance of these interactions in rRNA transcription regulation is supported by the isolation of $\Delta d k s A$ suppressor mutations in these domains ${ }^{25,26}$. The $\beta$ gate loop ( $\beta \mathrm{GL}$, residues $368-378)$ in the $\beta$ lobe domain contacts the T94, R99, and R103 residues of $\sigma$ region $1.2\left(\sigma_{1.2}\right.$, residues $\left.92-127\right)$ to enclose the RNAP cleft (Fig. $2 b$ ). The $\beta$ GL, $\sigma_{1.2}$, and $\sigma_{2.1}$ (residues $373-396$ ) contact the ntDNA strand of the discriminator from positions -8 to -6 (Fig. $2 c$ and Supplementary Movie 3); consistently, the $\beta \mathrm{GL}$ deletion and substitutions in $\sigma_{1.2}$ destabilize the $\mathrm{RPo}^{27,28}$. 
a
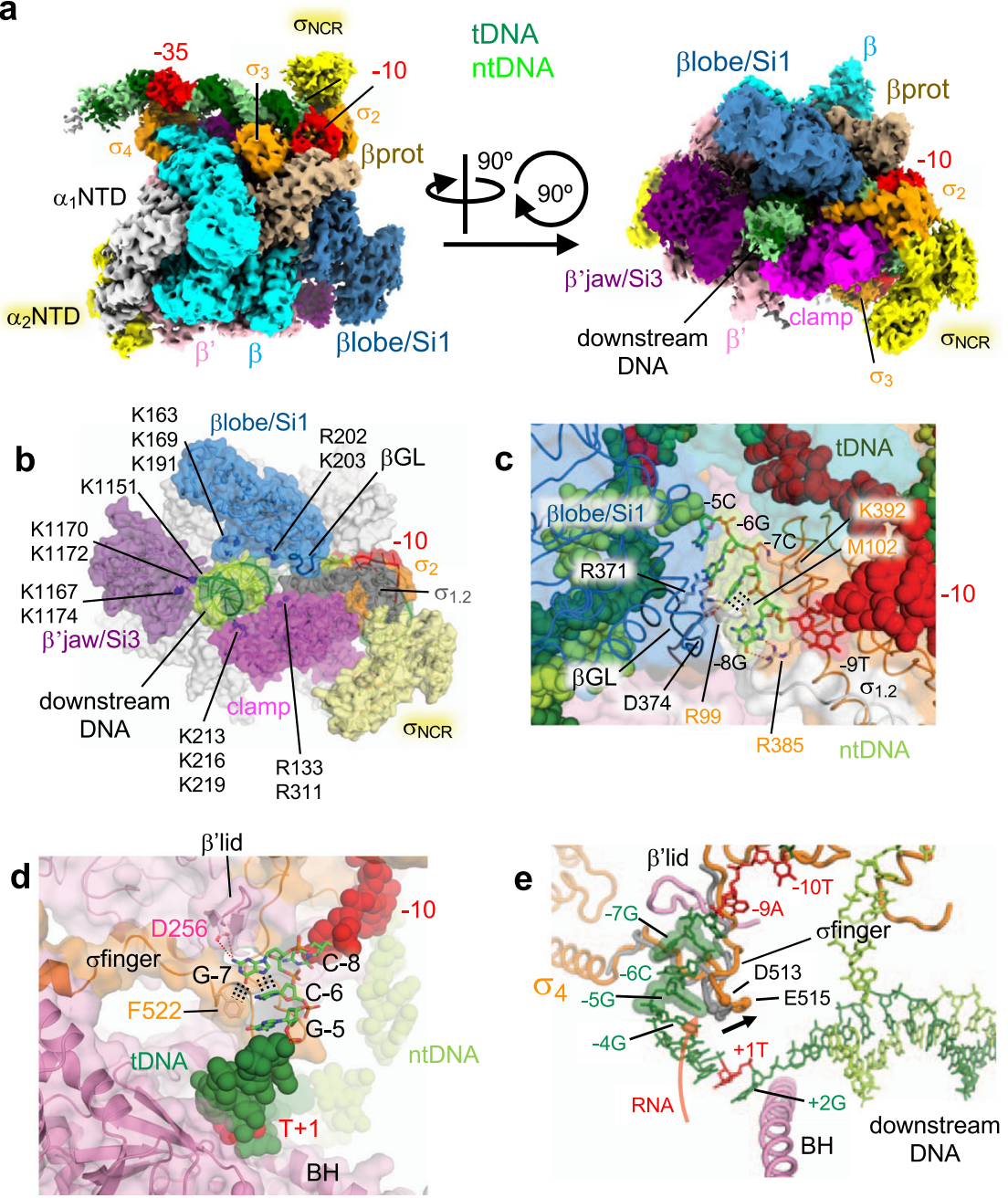

Fig. 2 Cryo-EM structure of the RNAP-rrnBP1 open complex (RPo). a Orthogonal views of the RPo cryo-EM density map. Subunits and domains of RNAP and DNA are colored and labeled the same as in Fig. 1b. b The structure of the RPo, highlighting basic residues of the $\beta$ lobe/Si1 (blue), $\beta^{\prime} j a w / S i 3$ (purple), and $\beta^{\prime}$ clamp (pink) interacting with downstream DNA (green) to stabilize the RPo. The structure is shown as a ribbon model with a transparent surface, and the basic residues are shown as spheres and labeled. $\mathbf{c}$ Close-up view of RNAP ( $\beta G L, \sigma_{1.1}$, and $\sigma_{1.2}$ ) and discriminator DNA (ntDNA) interaction. $\beta$ and $\sigma$ are depicted as ribbon models with transparent surfaces, and DNA is shown as CPK spheres. The G-8, C-7, and G-6 bases (stick model with transparent CPK spheres) that form salt bridges and Van del Waals interactions with residues from the $\beta G L$ and $\sigma_{1.2}$ (side chains shown as sticks; $\beta G L$ R371 and D374; $\sigma_{1.2}$ R99 and M102) are shown (depicted by red and black dashed lines). d Close-up view of the RNAP ( $\beta^{\prime}$ lid and ofinger) and discriminator DNA (tDNA) interaction. The G-7 base inserts into the pocket formed by the $\beta^{\prime}$ lid, ofinger and C- 6 base. $\beta^{\prime}$ and $\sigma$ are depicted as ribbon models with transparent surfaces, and DNA is shown as a stick model and CPK representation. The residues forming salt bridges and Van del Waals interactions with the G-7 base are shown (depicted by red and black dashed lines). e Comparison of the ofinger in RPo-rrnBP1 (this study, orange) and RPo-rpsTP2 ${ }^{14}$ (gray). The RPorrnBP1 structure is depicted as a cartoon (RNAP) and stick (DNA) models. When the rrnBP1 tDNA scrunches at the $-7 \mathrm{G}(\mathrm{t})$ position, $-5 \mathrm{G}(\mathrm{t})$ is located below the ofinger (orange), which shifts the ofinger position compared to that in nonscrunched RPo (gray). The ofinger dislocation (black arrow, $5 \AA$ at E515) makes additional space for RNA extension (red arrow).

The RNAP and $r r n B P 1$ complex starts RNA synthesis at the position 9 bp downstream from the -10 element $(+1 \mathrm{~A})$, which requires DNA scrunching ${ }^{29}$. The RPo structure revealed the path of the scrunched template strand, in which the G-7 base of tDNA fits into a pocket surrounded by the $\beta$ 'lid, ofinger ( $\sigma$ region 3.2) and the C-6 base (Fig. 2d, Supplementary Fig. $4 \mathrm{~b}$, e and Supplementary Movie 3). The importance of the G-7 base for rRNA expression is underscored by its conservation in all seven rRNA promoters in E. coli and rRNA promoters in other proteobacteria (Supplementary Fig. 1). Highly conserved D256 ( $\beta^{\prime}$ lid) and F522 (ofinger) residues form a salt bridge and Van der Waals interaction with the G-7 base, respectively. We found that alanine substitution of residue D256 ( $\beta^{\prime}$ lid) significantly stabilizes RNAP complexes with the rrnBP1 promoter (promoter complex half-life $t_{1 / 2}$ of $135 \pm 16 \mathrm{~s}$ vs. $34 \pm 7 \mathrm{~s}$ for wild-type
RNAP) (Fig. 3a, b). Similarly, a $\sigma$ finger deletion was shown to increase the stability of RNAP-rrnBP1 complex ${ }^{30}$, suggesting that the contacts of ofinger with tDNA may destabilize this promoter complex. The deletion of the $\sigma$ finger $^{30}$ or the G-7C substitution ${ }^{29}$ was also shown to shift the TSS to the $-3 \mathrm{~A}$ position, likely eliminating open complex scrunching. However, the D256A substitution did not change the TSS in $\operatorname{rrnBP} 1$, as revealed by primer extension analysis of the transcription products synthesized from $\mathrm{rrnBP} 1$ by wild-type and mutant RNAPs (Fig. 3c, lanes 3 and 4). Therefore, the contacts of the $\beta^{\prime}$ 'lid with G-7 may decrease the stability of the RNAP-rrnBP1 complex with scrunched DNA, possibly by favoring the unstable conformation of the template strand, without changing the TSS selection. The nontemplate DNA strand is also scrunched, making DNA bases around the single-stranded and double- 


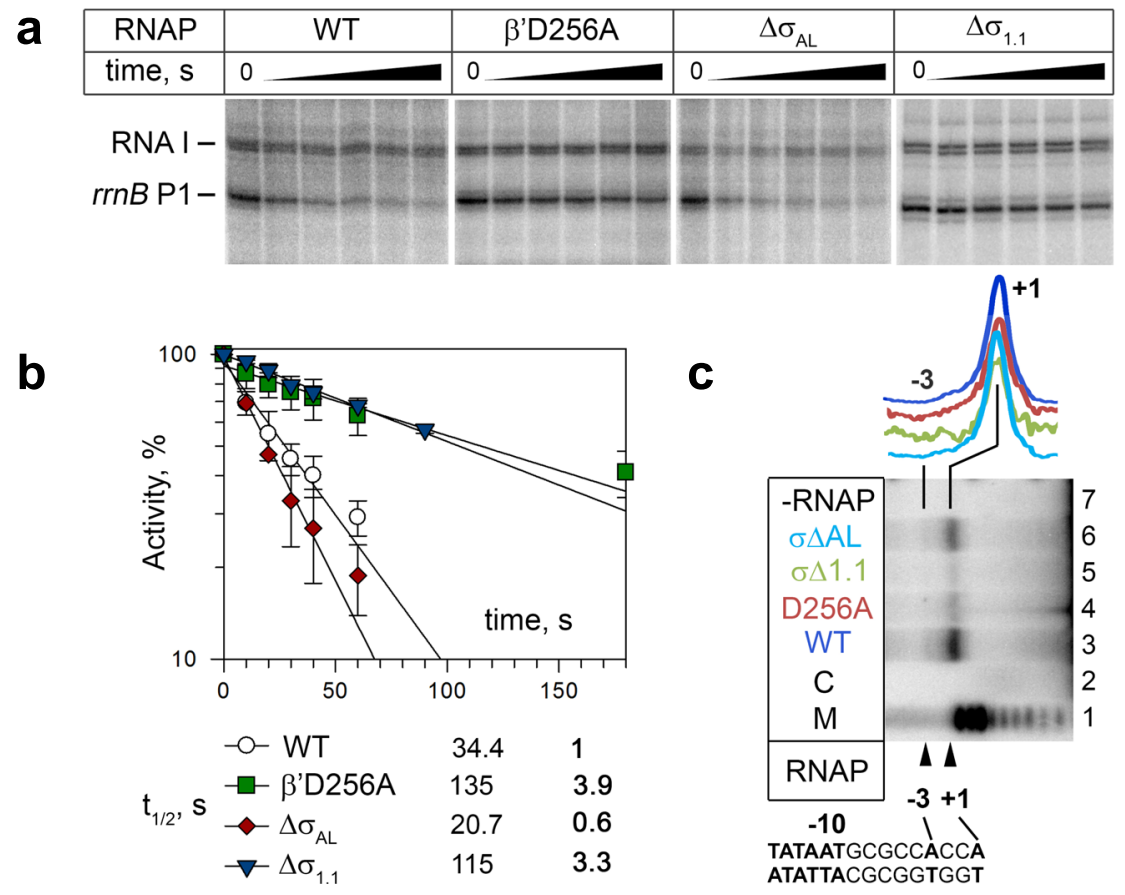

Fig. 3 Stabilities of rrnBP1 promoter complexes formed by wild-type and mutant RNAPs and their sensitivities against DksA/ppGpp. a Stabilities of rrnBP1 promoter complexes. Preformed complexes of E. coli RNAP with supercoiled plasmid DNA containing the rrnBP1 and RNAI promoters were incubated with competitor DNA (containing upstream fork-junction promoter DNA but lacking the TSS) for the indicated time intervals, followed by the addition of NTP substrates. Positions of the full-length transcripts are indicated. For each RNAP, the experiments were repeated independently two to five times with similar results. $\mathbf{b}$ Kinetics of promoter complex dissociation for wild-type and mutant RNAPs. The half-lives of the promoter complexes for each RNAP are shown (measured in the absence of DksA; mean values from two to five independent experiments). c Transcription start site mapping in rrnBP1 for wildtype and mutant RNAPs (a representative gel from two independent experiments). The products of in vitro transcription from rrnBP1 were used for primer extension with a $5^{\prime}$-labeled downstream primer, and the lengths of the products were compared with the starting primer (lane 2) and size markers (lane 1). Lane 7 shows a control reaction performed in the absence of RNAP. Positions of the transcription start sites in the scrunched $(+1)$ and relaxed $(-3)$ promoter complexes are indicated. The scanned profiles of the primer extension products adjusted by the intensity of the main product for each RNAP are shown above the gel. Source data are provided as a Source Data file.

stranded junction at the RNAP active site (from -2 to +2 positions) disordered.

Open complex scrunching may facilitate promoter escape of RNAP by reducing abortive RNA cycle ${ }^{29,31}$. Compared with RPorpsTP2 containing nonscrunched $\mathrm{DDNA}^{14}$, RPo-rrnBP1 shifts the ofinger $\sim 5 \AA$ away from tDNA, allowing accommodation of one additional base of RNA before its $5^{\prime}$-end reaches the ofinger (Fig. 2e). Since the ofinger is one of the major obstacles to promoter escape ${ }^{32-34}$, the partially displaced ofinger in the RPo may reduce the abortive RNA cycle or may prevent the formation of inactive moribund complexes ${ }^{31,35}$, promoting the robust expression of rRNA.

Cryo-EM structures of the RNAP and rrnBP1 promoter complex with DksA/ppGpp (RP-DksA/ppGpp). To reveal how $\mathrm{DksA} / \mathrm{ppGpp}$ binding to RNAP downregulates rRNA transcription, we visualized the RNAP, $r r n B P 1$, and $\mathrm{DksA} / \mathrm{ppGpp}$ complex (RP-DksA/ppGpp) by cryo-EM (Supplementary Table 1 and Supplementary Fig. 6). The classification of the cryo-EM data gave rise to two structures that differed mainly within the RNAP cleft; the first class shows the globular density corresponding to $\sigma_{1.1}$ (class I, RP1-DksA/ppGpp), and the second class shows the helical density corresponding to the downstream DNA (class II, RP2-DksA/ppGpp) (Fig. 4a, b and Supplementary Movie 4). In addition, the positions of $\beta$ lobe/Si1 are different in these classes (Fig. 4c).

In both classes, the cryo-EM density maps show ppGpp binding at sites 1 and 2 and DksA binding at the RNAP secondary channel (Supplementary Fig. 6e), as observed in the previous X-ray crystallography study ${ }^{11}$. DksA binds RNAP with its globular domain ( $G$ domain, contacts with the $\beta$ 'rim helix), coiled-coil tip (CC tip, contacts with the active site), CC (contacts with the bridge helix, the trigger loop, and linkers connecting to the $\beta^{\prime}$ Si3), and the C-terminal a helix (CT-helix, contacts with the $\beta$ lobe/SI1 domain) (Fig. 4a and Supplementary Movie 4). The CC of DksA prevents trigger helix formation and blocks NTP entry from the secondary channel, indicating that DksA must be displaced before RNAP initiates RNA synthesis ${ }^{11,36}$.

Both classes show the duplex DNA density from positions -42 to -14 (from the downstream edge of the UP element to the upstream edge of the -10 element) and also show the ssDNA density of the nontemplate strand of the -10 element (Supplementary Fig. 4). RP1-DksA/ppGpp retains $\sigma_{1.1}$ in the RNAP cleft, indicating that it represents an early stage intermediate during the closed to open complexes transition. While the transcription bubble is likely partially open in RP1DksA/ppGpp, the density of ntDNA from -5 to +20 and of tDNA from -13 to +20 is not traceable. Analysis of RP1DksA/ppGpp reveals a DksA/ppGpp-induced conformational change in $\beta$ lobe/Sil, $\beta$ 'jaw/Si3 and $\beta^{\prime}$ clamp, opening the downstream DNA cleft in RNAP and likely reducing the stability of RPo (Fig. 4d). The interactions of the ßlobe Si1 domain with DksA CT-helix and the conformational change of Sil were previously observed in the crystal structure of the RNAP-DksA/ppGpp complex ${ }^{11}$ but were smaller than in the cryo-EM structure, likely because of the crystal packing. 


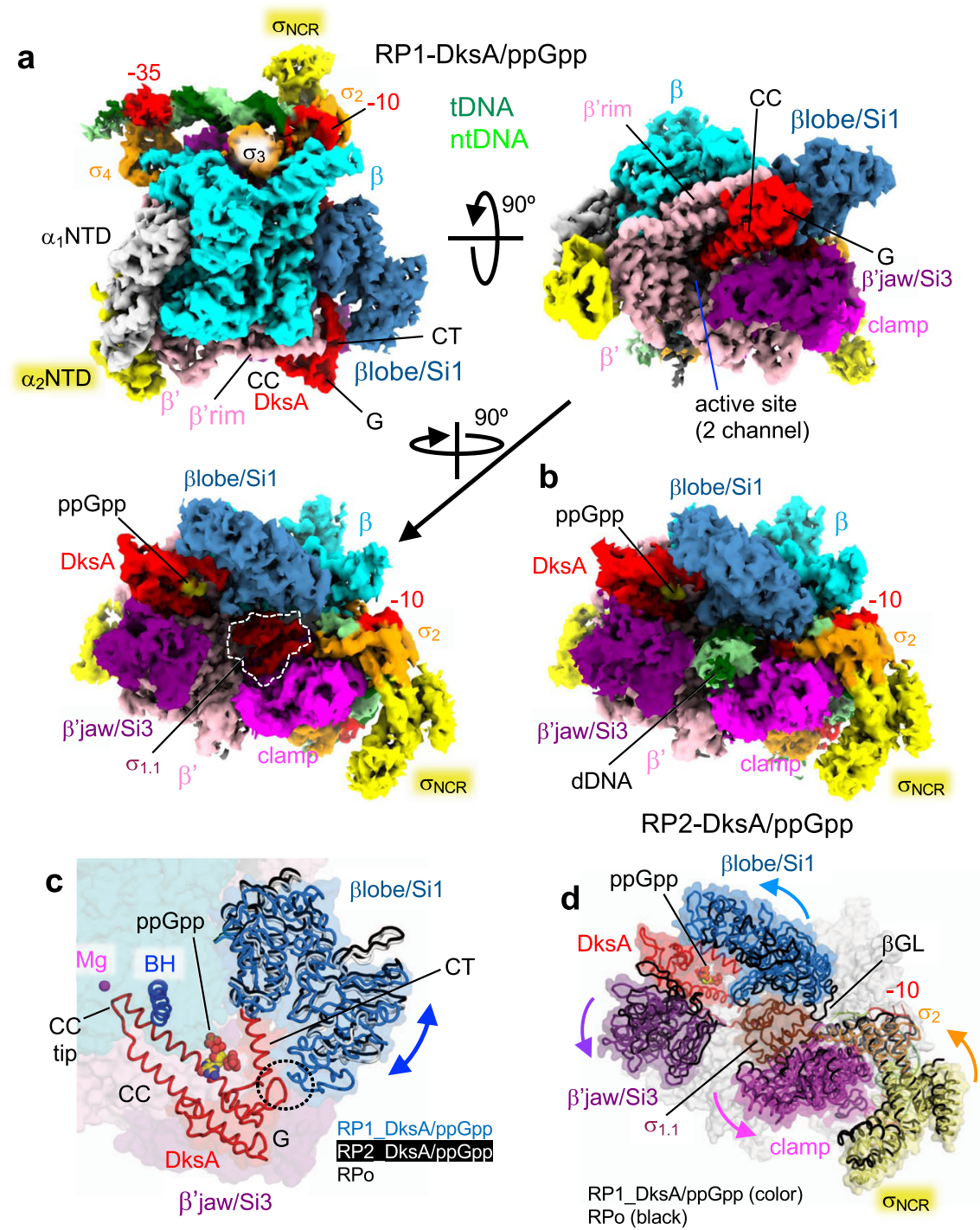

Fig. 4 Cryo-EM structures of the RNAP-rrnBP1 complex with DksA/ppGpp (RP-DksA/ppGpp). a Orthogonal views of the RP1-DksA/ppGpp cryo-EM density map. DNA, RNAP and DksA (G, G domain; CC, CC domain; CT, CT-helix) are indicated and colored. $\sigma_{1.1}$ is highlighted by a white dash. $\mathbf{b}$ The RP2DksA/ppGpp cryo-EM density map. The downstream DNA is accommodated in the RNAP cleft. c Close-up view of the $\beta$ lobe/Si1 conformational changes upon DksA binding, $\sigma_{1.1}$ ejection and downstream DNA binding. The structures of the $\beta$ lobe/Si1 in RP1-DksA/ppGpp (light blue), RP2-DksA/ppGpp (white), and RPo (black) are depicted as ribbon models with transparent surfaces and ribbon models ( $D k s A$, BH: bridge helix) of RP1-DksA/ppGpp. The interaction between $\beta$ lobe/Si1 and DksA in RP1-DksA/ppGpp is highlighted by a black dashed oval. d Conformational changes in the RNAP mobile domains upon binding of DksA/ppGpp. The structures of the RNAP mobile domains in RP1-DksA/ppGpp (colored) and RPo (black) are depicted as ribbon models with transparent surfaces of RP1-DksA/ppGpp.

The conformational change in $\beta$ lobe/Sil establishes a new contact with the DksA CT-helix, which is only observed in the cryo-EM structure (Fig. 4c and Supplementary Movie 4); the deletion of $\beta$ Sil was shown to reduce the DksA affinity to RNAP and impair its function ${ }^{37}$. Alanine substitution of an aspartate residue in the CT-helix directly involved in this interaction (D137A) decreases $r r n B P 1$ inhibition by DksA both in the absence and in the presence of ppGpp (Table 1).

The RP2-DksA/ppGpp complex contains downstream DNA (from +3 to +20 ) within the RNAP cleft, but the density of the DNA bubble (from -8 to +2 ) is not traceable (Fig. $4 \mathrm{~b}$ and Supplementary Fig. 4), suggesting that it represents a late-stage intermediate before forming the RPo. The $\sigma_{1.1}$ density is not traceable due to its ejection from the RNAP cleft. The conformations of $\beta$ lobe/Sil and $\beta$ 'clamp are akin to the RPo conformation, and the CT-helix of DksA does not contact with the Blobe/Si1 (Fig. 4c). Therefore, the transition between the two complexes may reduce the DksA affinity to RNAP and trigger its dissociation, which is an obligatory process to initiate RNA synthesis $^{11,36}$.

Ejection of $\sigma_{1.1}$ and conformational changes in $\sigma$ domain 2 during a RPc formation. The RPc structure revealed the ejection of $\sigma_{1.1}$ from the RNAP cleft and significant conformational changes in $\sigma$ domain 2 , including $\sigma_{1.2}$ and the nonconserved region between regions 1 and 2 ( $\sigma_{\mathrm{NCR}}$, residues $\left.128-372\right)$, in comparison with the apo-form holoenzyme RNAP ${ }^{38}$ and the RPo containing $r r n B P 1$ (this study).

From the apo-form to RPc, $\sigma_{1.2} / \sigma_{\mathrm{NCR}}$ of RNAP holoenzyme undergo a rigid rotation toward the clamp to establish contact with the $\beta^{\prime}$ clamp-toe ( $\beta^{\prime} \mathrm{CT}$, residues 143 to 180 ) (RPc, Fig. $5 \mathrm{a}$ ). Although this interaction was not observed in any previous structural study, it was predicted based on the biochemical/ genetic analysis of RNAP promoter escape and early elongation 
pausing ${ }^{39}$. It was shown that the interaction of the $\sigma_{\mathrm{NCR}}$ and $\beta^{\prime} \mathrm{CT}$ is important for promoter escape and hinders early elongation pausing, and amino acid substitutions at the interface modulate both processes (Supplementary Fig. 7).

The $\beta$ GL contacts the N-terminus of $\sigma_{1.2}$ to enclose the RNAP cleft in the apo-form RNAP, which prevents DNA loading (apo, Fig. 5a), but the same interaction in the RPo stabilizes the open

\begin{tabular}{|c|c|c|}
\hline \multirow[t]{2}{*}{ RNAP } & \multicolumn{2}{|l|}{$K_{\mathrm{d}, \mathrm{app}}(\mathrm{nM})$} \\
\hline & -ppGpp & ${ }^{+}$ppGpp \\
\hline WT & $\begin{array}{l}2200 \pm 430 \\
1\end{array}$ & $\begin{array}{l}860 \pm 180 \\
1\end{array}$ \\
\hline$\beta^{\prime} \mathrm{D} 256 \mathrm{~A}$ & $\begin{array}{l}4540 \pm 150 \\
2.1\end{array}$ & $\begin{array}{l}1640 \pm 220 \\
1.9\end{array}$ \\
\hline$\Delta \sigma_{\mathrm{AL}}$ & $\begin{array}{l}3000 \\
1.4\end{array}$ & $\begin{array}{l}1150 \pm 220 \\
1.3\end{array}$ \\
\hline$\Delta \sigma_{1.1}$ & $>10000$ & ND \\
\hline WT RNAP + & 3900 & $2440 \pm 1100$ \\
\hline DksA D137A & 1.8 & 2.8 \\
\hline
\end{tabular}

The apparent dissociation constants for DksA binding to promoter complexes $\left(K_{\mathrm{d}, \text { app }}\right)$ were calculated from the efficiency of transcription inhibition in titration experiments. The numbers in below $K_{d, a p p}$ values indicate fold changes in $K_{d, a p p}$ relative to the wild-type RNAP. complex bubble (Fig. 2b). In the case of RPc, the $\sigma_{1.2} / \sigma_{\mathrm{NCR}}$ rotation disrupts the $\beta \mathrm{GL}$ and $\sigma$ contact and widens the gap that allows the ejection of $\sigma_{1.1}$ and the entrance of discriminator DNA for the open complex bubble formation (RPc, Fig. 5a). Compared with the apo-form RNAP, the $\sigma_{\mathrm{NCR}}$ and $\beta^{\prime} \mathrm{CT}$ interaction in the RPc closes the $\beta$ 'clamp, which likely stimulates the ejection and prevents re-entry of $\sigma_{1.1}$ due to its steric clash with the $\beta$ ' clamp (Fig. 5b).

$\sigma_{\mathrm{NCR}}$ contains a highly negatively charged region (acidic loop, residues 167-213) (Supplementary Fig. 8a). Its conformation has not been determined due to its dynamic behavior, but since it is located near $\sigma_{2.3}$, it seems to prevent nonspecific DNA binding to $\sigma_{2.3}$ (Supplementary Fig. 8b). We speculate that after RNAP recognizes the UP and -35 elements, loading of the -10 element DNA onto $\sigma$ domain 2 triggers $\sigma_{\mathrm{NCR}}$ rotation due to chargecharge repulsion. After DNA unwinds around the -10 element, $\sigma_{\mathrm{NCR}}$ returns to its position, as seen in the RPo akin to the apoform RNAP, and may enhance the electrostatic interaction between $\sigma_{2}$ and -10 element DNA (Supplementary Fig. 8c). Consistently, deletion of the acidic loop $\left(\Delta \sigma_{\mathrm{AL}}\right)$ had a weak destabilizing effect on the rrnBP1-RNAP complex, without strong effects on DksA inhibition (Fig. 3 and Table 1).

In addition to the promoter DNA bound on the holoenzyme surface of RPc, a second DNA molecule was accommodated in the RNAP cleft, akin to the downstream DNA of the RPo. We argue that the double-stranded DNA binding in the RNAP cleft is the consequence but not the cause of $\sigma_{1.1}$ ejection and the
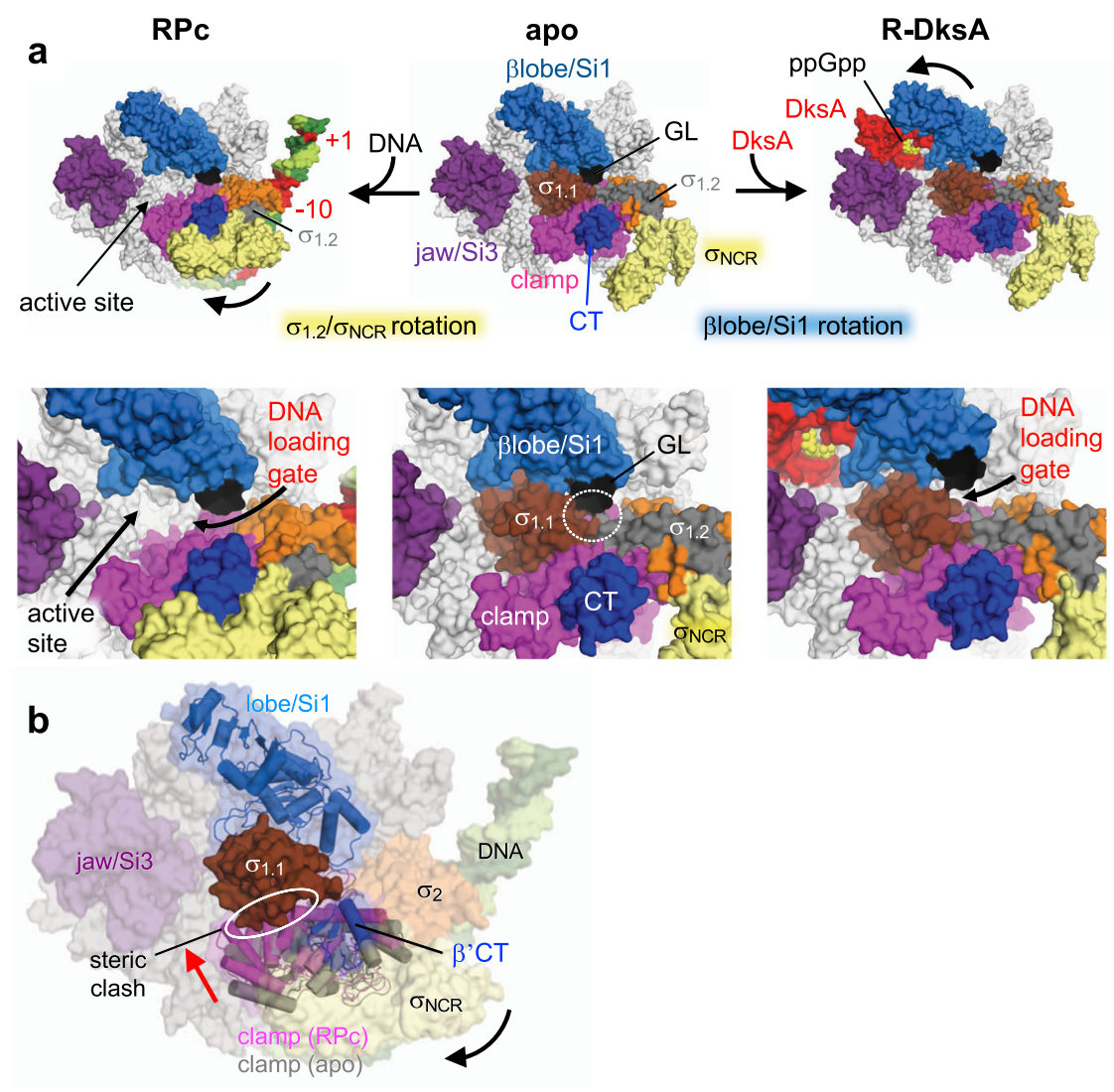

Fig. 5 Opening the DNA loading gate by moving the $\boldsymbol{\sigma}_{\mathbf{1 . 2}} / \boldsymbol{\sigma}_{\mathbf{N C R}}$ or $\boldsymbol{\beta}$ lobe/Si1 domain. a Comparison of the $\sigma_{1.2} / \sigma_{\mathrm{NCR}}$ and $\beta$ lobe/Si1 conformations in apoRNAP (middle), RPc (left), and RNAP-DksA/ppGpp (R-DksA, right, DNA is removed from the RP1-DksA/ppGpp to model this complex). RNAP (subunits and domains), DksA and DNA are indicated. Close-up views of the RNAP cleft are shown below. The DNA loading gate is closed in the apo-RNAP due to the $\beta$ gate loop $\left(\beta \mathrm{GL}\right.$ ) contacts with $\sigma_{1.1} / \sigma_{1.2}$ (white dashed oval). The opening of the DNA loading gate in RPc and RP1-DksA/ppGpp is indicated by blue and red arrows, respectively. $\mathbf{b}$ A proposed model of $\sigma_{1.1}$ ejection in the RPc. The RPc is depicted as a transparent surface with cartoon models of the clamp (purple) and the lobe/Si1 (blue). The clamp in the apo-form RNAP and the lobe/Si1 in RP1-DksA/ppGpp are colored gray and white, respectively. In the $\mathrm{RPC}$, the $\sigma_{\mathrm{NCR}}$ rotation (black arrow) contacts the $\beta^{\prime} \mathrm{CT}$, resulting in clamp movement toward $\sigma_{1.1}$ (red arrow) and a steric clash with $\sigma_{1.1}$ (white oval). 
$\sigma_{1.2} / \sigma_{\mathrm{NCR}}$ changes. First, the presence of $\sigma_{1.1}$ at the RNAP cleft in the apo-holoenzyme prevents any DNA binding at this cleft; therefore, $\sigma_{1.1}$ ejection has to be completed prior to binding of DNA and it requires disruption of the $\beta G L$ and $\sigma$ contacts coupled to the $\sigma_{\mathrm{NCR}}$ rotation. Second, none of the available promoter complex cryo-EM structures containing doublestranded DNA at the RNAP cleft show the $\sigma_{\mathrm{NCR}}$ rotation ${ }^{14,40}$ suggesting that the downstream DNA binding by itself is unlikely to trigger the conformational change in $\sigma_{1.2} / \sigma_{\mathrm{NCR}}$. Thus, the RPc structure illustrates how the downstream DNA cleft in RNAP becomes prepared for binding of the downstream DNA duplex during open complex formation.

DksA/ppGpp binding to RNAP also partially opens the DNA loading gate by moving the $\beta$ lobe/Sil away from $\sigma_{1.1} / \sigma_{1.2}$, but it is not strictly coupled to the $\sigma_{1.1}$ ejection from the RNAP cleft (R-DksA, Fig. 5a). Similarly, the structures of the RNAP-TraR complex and several RNAP-DNA complex intermediates prepared in the presence of TraR also showed the opening of the DNA loading gate by shifting the $\beta$ lobe/Si1 position but did not show $\sigma_{1.2} / \sigma_{\mathrm{NCR}}$ rotation. Furthermore, $\sigma_{1.1}$ was not ejected from the RNAP cleft at the stage of RPc formation (Supplementary Fig. 9a) ${ }^{14,38}$.

To understand the role of $\sigma_{1.1}$ in rRNA transcription, we characterized an RNAP derivative lacking $\sigma_{1.1}\left(\Delta \sigma_{1.1}\right.$-RNAP) in terms of its $r r n B P 1$ transcription activity and sensitivity to DksA. Compared to the wild-type (WT) RNAP, $\Delta \sigma_{1.1}$-RNAP has an increased $r r n B P 1$ complex stability, both in the absence of DksA (increase in $t_{1 / 2}$ from $34 \mathrm{~s}$ to $115 \mathrm{~s}$ ) and in its presence (increase in $\mathrm{t}_{1 / 2}$ from $\ll 10 \mathrm{~s}$ to $20 \mathrm{~s}$ ) (Fig. $3 \mathrm{a}, \mathrm{b}$ ), and decreased sensitivity to DksA (Table 1). At the same time, the $\Delta \sigma_{1.1}$ deletion (and, similarly, $\Delta \sigma_{\mathrm{AL}}$ ) does not change the transcription start site in $\operatorname{rrnBP} 1$ suggesting that $\sigma_{1.1}$ does not affect DNA scrunching during transcription initiation (Fig. 3c). Overall, the results indicate that $\sigma_{1.1}$ plays an important role in the destabilization of rRNA promoter complexes and their regulation by DksA/ppGpp.

\section{Discussion}

Mechanism of rRNA-specific transcription inhibition by DksA/ ppGpp. Structural and biochemical studies of bacterial RNAP transcription suggest that the order of DNA loading around the TSS and DNA opening may be interchangeable during promoter recognition (i.e., DNA melts first outside RNAP (melt-load) or DNA melts after loading inside the RNAP cleft (load-melt)) depending on $\sigma$ factors, promoters, transcription factors and conditions $15,33,41,42$. By combining structural and biochemical data from this and previous studies, we propose two pathways of RPo formation (Fig. 6 and Supplementary Movie 5). We hypothesize that RNAP may use alternative mechanisms of RPo formation with $r r n B P 1$ and possibly other promoters, requiring the opening of the DNA loading gate (disrupting the $\beta$ GL contact to $\sigma), \sigma_{1.1}$ ejection from the DNA binding channel, and unwinding of the -10 element plus discriminator DNA, depending on the absence or presence of DksA/ppGpp.

Without DksA/ppGpp (top, RPo formation), free RNAP (R) binds promoter DNA (RPc), which opens the DNA loading gate by ejecting $\sigma_{1.1}$ from the RNAP cleft, making RNAP competent for melting and loading discriminator DNA (RPi) into the RNAP cleft, which results in efficient RPo formation. The scrunched open complex (RPo) releases RNAP from the rRNA promoter rapidly to proceed with RNA synthesis (EC).

In the presence of DksA/ppGpp (bottom, RPo formation with DksA/ppGpp), DksA/ppGpp binding to RNAP rotates the $\beta$ lobe/ Sil to DksA, which partially opens the DNA loading gate by disrupting the interaction between GL and $\sigma$ (R-DksA). However, $\sigma_{1.1}$ ejection is uncoupled from RPc formation (RPc-DksA), and $\sigma_{1.1}$ can remain inside the RNAP cleft until the late stages of the open complex formation (RPi-DksA). This pathway favors the melt-load model for RPo formation (RPo-DksA), in which DNA is accommodated above the $\beta$ lobe domain and unwinds outside the RNAP cleft (Supplementary Fig. 9b) followed by singlestranded tDNA entry into the active site of RNAP ${ }^{14}$. DNA unwinding outside the RNAP cleft is unfavorable in the DksA/ ppGpp-free RNAP due to a steric clash of the discriminator DNA with the $\beta$ lobe. The progression of DNA unwinding from the -10 element to the TSS is energetically less favorable for DksA/ ppGpp-sensitive promoters (e.g., $r r n B P 1$ and $r p s T P 2)$ containing the $\mathrm{G}+\mathrm{C}$-rich discriminator than for less DksA/ppGpp-sensitive promoters (e.g., T7A1 and RNA1) containing an $\mathrm{A}+\mathrm{T}$ rich discriminator (Supplementary Fig. 10). E. coli promoters that are sensitive to DksA/ppGpp contain $\mathrm{G}+\mathrm{C}$-rich discriminators ${ }^{43}$. Replacing the $\mathrm{A}+\mathrm{T}$-rich discriminator of the uspA promoter, which is positively regulated by DksA/ppGpp, with the one from the $r r n B P 1$ promoter makes the $u s p A$ hybrid promoter sensitive to $\mathrm{DksA} / \mathrm{ppGpp}^{44}$, indicating that discriminator sequences play an important role in responding to DksA/ppGpp. Although DksA could inhibit transcription regardless of the promoter bound to RNAP, by inhibiting NTP entry and folding trigger helix, stable promoter complex formation decreases DksA binding to RNAP, thus relieving the inhibition ${ }^{11,36}$. The completion of discriminator DNA loading into the RNAP cleft can likely occur not only in DksA/ppGpp-insensitive promoters but also in a fraction of the rRNA promoter complexes to maintain a basal level of rRNA expression under stress growth conditions. This likely pushes the Blobe/Sil away from the CT-helix of DksA (RPo-DksA), allowing rapid dissociation of DksA from the RNAP secondary channel (RPo) followed by the initiation of transcription (EC).

From this study, we proposed two alternative pathways for opening the gate of the DNA binding channel of RNAP depending on the absence or presence of DksA/ppGpp (Fig. 6 and Supplementary Movie 5) and shed light on the functions of $\sigma_{1.1}$, $\sigma_{1.2}, \sigma_{\mathrm{NCR}}$, and $\beta$ lobe/Si1 domains to explain how DksA/ppGpp specifically inhibits rRNA transcription. Intriguingly, DksA/ ppGpp is able to activate transcription at some $\sigma^{70}$-promoters ${ }^{45}$ and promoters recognized by alternative $\sigma$ factors, including $\sigma^{S 46}$ and $\sigma^{\mathrm{E} 47}$. Neither $\sigma^{S}$ nor $\sigma^{\mathrm{E}}$ contains $\sigma_{1.1}$ or $\sigma_{\mathrm{NCR}}$, and the $\sigma^{\mathrm{S}}$ and $\sigma^{\mathrm{E}}$ holoenzymes use the $\beta \mathrm{GL}$ to close the DNA loading gate ${ }^{48,49}$. DNA binding to the $\sigma$ domain 2 of $\sigma^{\mathrm{S}}$ or $\sigma^{\mathrm{E}}$ cannot facilitate the opening of the DNA loading gate, as described in the case of the $\sigma^{70}$ holoenzyme (Fig. 6, top). However, DksA/ppGpp binding followed by the movement of the Klobe/Sil domain could still open the DNA loading gate of these RNAP holoenzymes as described above (Fig. 6, bottom), possibly explaining the stimulatory effects of DksA/ppGpp on transcription from some $\sigma^{\mathrm{S}}$ - and $\sigma^{\mathrm{E}}$-dependent promoters. Further structural analyses of the $\sigma^{70}$, $\sigma^{\mathrm{S}}$, and $\sigma^{\mathrm{E}}$ RNAP promoter complexes with DksA/ppGpp together with their biochemical characterization will be needed to complete our understanding of DksA/ppGpp-dependent transcription regulation.

\footnotetext{
Methods

Preparation of rrnBP1 DNA. The $r r n B P 1$ promoter DNA was synthesized (IDT) according to the native $r r n B P 1$ sequence and annealed in a $40 \mu \mathrm{L}$ reaction mixture containing $10 \mathrm{mM}$ Tris- $\mathrm{HCl}(\mathrm{pH} 8.0), 50 \mathrm{mM} \mathrm{NaCl}$, and $1 \mathrm{mM}$ EDTA to a final concentration of $0.5 \mathrm{mM}$. The solution was heated at $95^{\circ} \mathrm{C}$ for $10 \mathrm{~min}$, and then the temperature was gradually decreased to $22^{\circ} \mathrm{C}$. The sequence of the nontemplate strand is $5^{\prime}$-CAGAAAATTATTTTAAATTTCCTCTTGTCAGGCCGGAATA ACTCCCTATAATGCGCCACCACTGACACGGACTCTACGAG-3'. The transcription start site is underlined, and the template sequence is $5^{\prime}$-CTCGTAGAGTC CGTGTCAGTGGTGGCGCATTATAGGGAGTTATTCCGGCCTGACAAGAGG AAATTTAAAATAATTTTCTG-3'.
} 


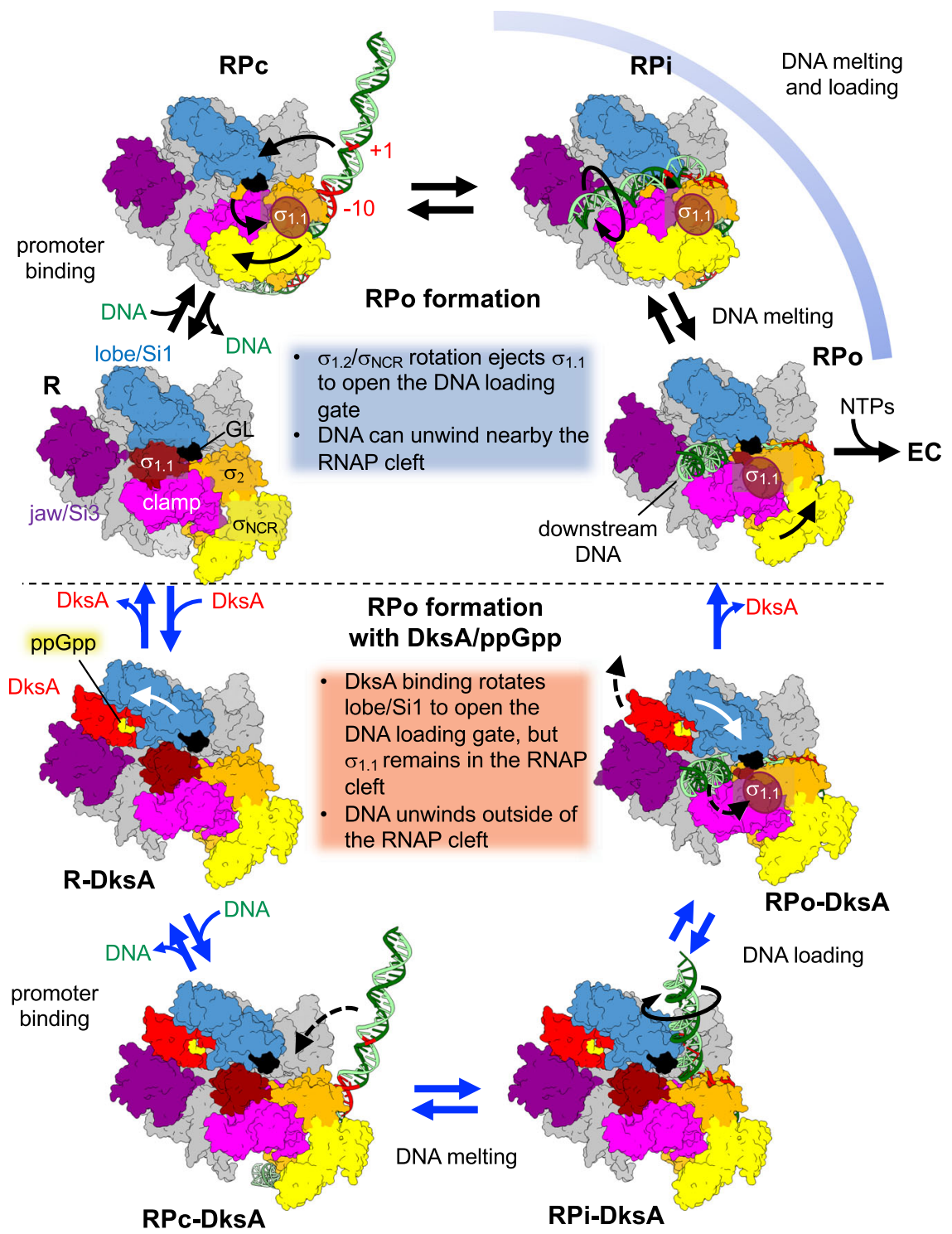

Fig. 6 Alternative pathways for open promoter complex formation. Two distinct pathways are shown for open promoter complex formation without (top, blue caption) and with DksA/ppGpp (bottom, red caption). The RNAP holoenzyme (clamp, pink; $\sigma_{\mathrm{NCR}}$, yellow; $\sigma_{1.1}$, brown; $\sigma_{2}$, orange; $\beta$ lobe/Si1, light blue; jaw/Si3, purple; rest of RNAP, gray), promoter DNA (tDNA, dark green; ntDNA, light green), DksA (red), and ppGpp (yellow) are shown. Only RPi is a hypothetical intermediate, but others (RPc, RPo, R-DksA, RPc-DksA, RPi-DksA, and RPo-DksA) represent the structures determined in this and previous studies. EC elongation complex.

Cryo-EM sample preparation. E. coli $\sigma^{70}$ RNAP holoenzyme and DksA were purified, as described previously 11,50 . To prepare the RNAP and $r r n B P 1$ promoter complex, E. coli $\sigma^{70}$ RNAP $(20 \mu \mathrm{M})$ and $r r n B P 1$ promoter DNA $(40 \mu \mathrm{M})$ were preincubated for $5 \mathrm{~min}$ at $37^{\circ} \mathrm{C}$ in buffer $(10 \mathrm{mM}$ HEPES, pH 8.0, $50 \mathrm{mM} \mathrm{NaCl}$, $0.1 \mathrm{mM}$ EDTA, $5 \mathrm{mM}$ DTT and $\left.5 \mathrm{mM} \mathrm{MgCl}_{2}\right)$. This sample was used for determining the cryo-EM structure of RPo (Supplementary Fig. 3). To stabilize the RPo, NTPs (ATP and a nonhydrolyzable CMPCPP (cytidine- $5^{\prime}-[(\alpha, \beta)$-methyleno]triphosphate, Jena Bioscience) ( $2 \mathrm{mM}$ each) were added to the RNAP-rrnBP1 promoter complex; however, this condition formed the RPc and RPtic (Supplementary Fig. 2) as described in "Results".

To prepare RP-DksA/ppGpp (Supplementary Fig. 6), E. coli $\sigma^{70}$ RNAP $(20 \mu \mathrm{M})$ was preincubated with a fivefold molar excess of DksA $(100 \mu \mathrm{M})$ and ppGpp $(2 \mathrm{mM})$ for $5 \mathrm{~min}$ at $37^{\circ} \mathrm{C}$ in the buffer $(10 \mathrm{mM}$ HEPES, pH 8.0, $50 \mathrm{mM} \mathrm{NaCl}$, $0.1 \mathrm{mM}$ EDTA, $5 \mathrm{mM} \mathrm{DTT}$, and $5 \mathrm{mM} \mathrm{MgCl}_{2}$ ). The $r r n B P 1$ promoter DNA $(40 \mu \mathrm{M})$ was added to the reaction and further incubated for 5 mins at $37^{\circ} \mathrm{C}$. Before freezing the grids, $8 \mathrm{mM}$ CHAPSO (Hampton research) was added to the reaction. A $3.5 \mu \mathrm{L}$ sample was applied to a glow-discharged C-Flat Holey Carbon grid (Cu 2/1, 400 mesh), blotted and plunge-frozen in liquid ethane using a Vitrobot Mark IV (FEI, USA) with $95 \%$ humidity at $4{ }^{\circ} \mathrm{C}$.

Cryo-EM data acquisition. The grid was imaged using a $300 \mathrm{keV}$ Titan Krios (Thermo Fisher) microscope equipped with a K3 direct electron detector (Gatan) and controlled by the Latitude S (Gatan, Inc.) software at the National Cancer Institute's Cryo-EM Facility at Frederick. The defocus range was -1.0 to $-3.0 \mu \mathrm{m}$ and the magnification was $\times 81,000$ in electron counting mode (pixel size $=1.08 \AA$ / pixel). Forty frames per movie were collected with a dose of $1.125 \mathrm{e}^{-} / \AA^{2} /$ frame, giving a total dose of $45 \mathrm{e}^{-} / \AA^{2}$.

Cryo-EM data processing. The RNAP-rrnBP1 complex with ATP/CMPCPP data was processed using Relion3.0.8 ${ }^{51}$. A total of 8315 movies were collected, aligned, and dose weighted using MotionCor $2^{52}$. CTF fitting was performed with Gctf ${ }^{53}$. Initially, $\sim 1000$ particles were manually picked to generate particle templates followed by automated picking, resulting in a total of 1,449,010 particles subjected to $2 \mathrm{D}$ classification. From the 2D classes, 1,442,810 particles were chosen for the $3 \mathrm{D}$ classification to four classes. Poorly populated classes were removed, resulting in datasets of 541,257 (37\%) particles for the first class (RPc) and 464,512 (32\%) particles for the second class. The first class was further $3 \mathrm{D}$ classified without alignments twice to further clean the data, resulting in datasets of 67,187 particles. The particles were refined and postprocessed to generate the density map at $4.14 \AA$ resolution. The resolution of the density map of the second class was $3.53 \AA$.

The RNAP-rrnBP1 complex data were processed using Relion3.0.8. A total of 4748 movies were collected, aligned and dose weighted using MotionCor2. CTF fitting was performed with Gctf. Approximately 1000 particles were manually picked to generate particle templates followed by automated picking, resulting in a 
total of 563,500 particles. Particles were 2D classified, and 561,753 particles were chosen for the 3D classification. Of the four 3D classes, class 1 (RPo) was the most populated class $(349,752$ particles, $62 \%)$ and was autorefined. The map was postprocessed to give a structure of RPo at $3.53 \AA$.

The RP-DksA/ppGpp complex data were processed using cryoSPARC V2.9.0 ${ }^{54}$. A total of 4,926 movies were collected, and the movies were aligned, and dose weighted using Patch-motion correction. CTF fitting was performed with PatchCTF estimation. Initially, $\sim 1000$ particles were manually picked to generate particle templates followed by automated picking, resulting in a total of 418,049 particles subjected to 2D classification. After two rounds of 2D classification to remove junk particles, 361,048 particles were used to generate two ab initio models. Junk particles were removed, resulting in a dataset of 275,629 particles chosen for the 3D classification (heterogenous refinement). Poorly populated classes were removed, resulting in a dataset of 49,995 particles to generate the density map at $3.62 \AA$ resolution for the first class (RP1-DksA/ppGpp) and a dataset of 79,275 particles to generate the density map at $3.58 \AA$ resolution for the second class (RP2-DksA/ ppGpp). The particles were 3D autorefined without the mask and postprocessed (homogenous refinement)

Structure refinement and analysis. To refine the closed and open complex structures, the E. coli RNAP holoenzyme crystal structure (PDB: 4YG2) was manually fit into the cryo-EM density map using Chimera ${ }^{55}$ and real-space refined using Phenix ${ }^{56}$. In the real-space refinement, the domains of RNAP were rigidbody refined and then subsequently refined with secondary structure, Ramachandran, rotamer, and reference model restraints. To refine the structures of RP1DksA/ppGpp and RP2-DksA/ppGpp, E. coli RNAP and DksA/ppGpp complex crystal structures (PDB: 5VSW) were manually fit into the cryo-EM density map using Chimera. DNA was manually built by using $\operatorname{Coot}^{57}$. The structure was refined by the same method as the closed and open complex structures. To superpose the structures of RNAP, we used the aNTDs and the catalytic domains (DPBB domains from the $\beta$ and $\beta$ ' subunits) of RNAP as references. Figures were prepared by ChimeraX ${ }^{58}$ and Pymol.

\section{Preparation of RNAP and transcription factors for in vitro transcription} Mutant variants of RNAP, $\sigma^{70}$, and DksA were obtained by site-directed mutagenesis. The sequences of all primers used in this study are shown in Supplementary Table 2 . The D256A substitution in the $\beta^{\prime}$ subunit was obtained in pVS10 encoding all RNAP subunits, with the $r p o C$ gene containing a C-terminal $\mathrm{His}_{6^{-}}$ $\operatorname{tag}^{59}$. The $\sigma^{70}$ and DksA variants containing an $\mathrm{N}$-terminal $\mathrm{His}_{6}$-tag were cloned into pET28. To obtain $\sigma \Delta_{1.1}$, the $5^{\prime}$-terminal part of the $r p o D$ gene encoding residues 2-94 was deleted. To obtain $\sigma \Delta_{\mathrm{AL}}$, codons 168-212 were replaced with three glycine codons. All proteins were expressed in E. coli BL21(DE3). The wildtype and mutant core RNAPs were purified using Polymin P precipitation followed by heparin (HiTrap Heparin column), Ni-affinity (HisTrap HP column), and anion exchange (MonoQ column) chromatography steps (all columns from GE Healthcare $)^{59}$. The wild-type and mutant $\sigma^{70}$ factors were purified from inclusion bodies with subsequent renaturation and Ni-affinity chromatography ${ }^{34}$. The $\sigma \Delta_{1.1}$ protein was subjected to thrombin protease (GE Healthcare) treatment in PBS buffer (10 h of incubation at $4{ }^{\circ} \mathrm{C}$ with ten units of protease per $\mathrm{mg}$ of protein), followed by incubation with Ni-NTA agarose (GE Healthcare) to remove the His-tag and Histagged thrombin. To purify DksA, bacterial pellet from 0.5 liters of cell culture was resuspended in $25 \mathrm{ml}$ of lysis buffer $(50 \mathrm{mM}$ Tris- $\mathrm{HCl}, \mathrm{pH} 7.9,250 \mathrm{mM} \mathrm{NaCl}$, $10 \mathrm{mM}$ EDTA, $0.5 \mathrm{mM}$ phenylmethylsulfonyl fluoride, $1 \mathrm{mM}$ 2-mercaptoethanol, $0.1 \mathrm{mM} \mathrm{ZnCl}_{2}$ ) and lysed using a French press. The supernatant obtained after centrifugation was loaded onto a 5-ml HiTrap chelating column (GE Healthcare) charged with $\mathrm{Ni}^{2+}$ and equilibrated with loading buffer (10 mM Tris- $\mathrm{HCl}, \mathrm{pH} 7.9$, $500 \mathrm{mM} \mathrm{NaCl}, 0.5 \mathrm{mM}$ 2-mercaptoethanol, $0.1 \mathrm{mM} \mathrm{ZnCl}_{2}$ ). The column was washed with the same buffer containing $60 \mathrm{mM}$ imidazole, and DksA was eluted with buffer containing $300 \mathrm{mM}$ imidazole and dialyzed overnight against $50 \mathrm{mM}$ Tris- $\mathrm{HCl}, 300 \mathrm{mM} \mathrm{NaCl}, 1 \mathrm{mM}$ DTT, and $0.1 \mathrm{mM} \mathrm{ZnCl}_{2}$. Glycerol was added to $50 \%$, and aliquots were stored at $-70^{\circ} \mathrm{C}$.

Transcription in vitro. Analysis of transcription in vitro was performed using a supercoiled pTZ19 template containing rrnBP1 cloned $88 \mathrm{nt}$ upstream of the his terminator; the second transcript monitored in the assays was $110 \mathrm{nt}$ RNAI encoded by the ori region of the plasmid ${ }^{30}$. For measurements of promoter complex stabilities, promoter complexes were prepared by mixing core RNAP (100 nM final concentration) with wild-type or mutant $\sigma^{70}$ factors $(250 \mathrm{nM})$ in transcription buffer ( $40 \mathrm{mM}$ Tris- $\left.\mathrm{HCl}, \mathrm{pH} 7.9,10 \mathrm{mM} \mathrm{MgCl}_{2}, 40 \mathrm{mM} \mathrm{KCl}\right)$ and supercoiled plasmid DNA (10 nM), followed by incubation for $7 \mathrm{~min}$ at $37^{\circ} \mathrm{C}$. DksA and ppGpp were added at $2 \mu \mathrm{M}$ and $200 \mu \mathrm{M}$, respectively, when indicated. An upstream fork-junction competitor DNA was added (template strand 5'-ACGAGCCGGAAGCAT-3', nontemplate strand 5'-ATGCTTCCGGCTCGTATAATGTGTGGAA- $3^{\prime}$; the -10 sequence is underlined) to $2 \mu \mathrm{M}$, and the samples were incubated at $37^{\circ} \mathrm{C}$ for the indicated time intervals. NTP substrates were added to final concentrations of $200 \mu \mathrm{M}$ ATP, CTP, GTP, and $10 \mu \mathrm{M}$ UTP, with the addition of $\left.\alpha-{ }^{32} \mathrm{P}\right]-\mathrm{UTP}$ together with rifapentin $(5 \mu \mathrm{g} / \mathrm{ml})$ to prevent re-initiation. The reactions were stopped after $5 \mathrm{~min}$ with $8 \mathrm{M}$ urea and $20 \mathrm{mM}$ EDTA, and RNA products were separated by $15 \%$ denaturing PAGE, followed by phosphorimaging using a Typhoon 9500 scanner (GE Healthcare). To calculate the observed half-life times for promoter complex dissociation $\left(t_{1 / 2}\right)$, the data were fitted to the oneexponential equation $A=A_{0} \times \exp \left(-\mathrm{t} \times k_{\mathrm{obs}}\right)$, where $\mathrm{A}$ is the RNAP activity at a given time point after competitor addition, $\mathrm{A}_{0}$ is the activity measured in the absence of the competitor, $k_{\mathrm{obs}}$ is the observed rate constant, and $t_{1 / 2}=\ln 2 / k_{\mathrm{obs}}$.

For measurements of apparent DksA affinities, promoter complexes were prepared in the same way with $50 \mathrm{nM}$ core RNAP, $250 \mathrm{nM} \sigma^{70}(250 \mathrm{nM})$, and $2 \mathrm{nM}$ supercoiled plasmid DNA in transcription buffer containing $100 \mu \mathrm{g} / \mathrm{ml} \mathrm{BSA}$ for $7 \mathrm{~min}$ at $37^{\circ} \mathrm{C}$, followed by the addition of DksA (from $10 \mathrm{nM}$ to $10 \mu \mathrm{M}$ ), either in the absence or in the presence of ppGpp $(100 \mu \mathrm{M})$. Transcription was performed for $15 \mathrm{~min}$ at $37^{\circ} \mathrm{C}$ with $200 \mu \mathrm{M}$ ATP, CTP, GTP, and $10 \mu \mathrm{M}$ UTP (plus $\alpha-\left[{ }^{32} \mathrm{P}\right]-\mathrm{UTP}$ ), and RNA products were analyzed as described above. The apparent dissociation constant values $\left(K_{\mathrm{d} \text {,app }}\right)$ were calculated from the hyperbolic equation: $\mathrm{A}=\mathrm{A}_{\max } \times\left(1-[\mathrm{DksA}] /\left(K_{\mathrm{d}, \mathrm{app}}+[\mathrm{Dks} \mathrm{A}]\right)\right)$, where $\mathrm{A}$ is the observed RNAP activity and $A_{\max }$ is the RNAP activity measured in the absence of DksA.

For transcription start site mapping, in vitro transcription was carried out with wild-type and mutant RNAPs ( $100 \mathrm{nM}$ core enzyme, $\left.500 \mathrm{nM} \sigma^{70}\right)$ in the transcription buffer for $15 \mathrm{~min}$ at $37^{\circ} \mathrm{C}$ with the pTZ19rrnBP1 plasmid $(25 \mathrm{nM})$ in the presence of $200 \mu \mathrm{M}$ ATP, GTP, CTP and $20 \mu \mathrm{M}$ UTP. RNA was ethanol precipitated, dissolved in water, mixed with $1 \mathrm{pmol}$ of $5^{\prime}-{ }^{32} \mathrm{P}$-labeled primer (corresponding to positions from +30 to +10 of the initially transcribed region in rrnBP1), incubated at $65^{\circ} \mathrm{C}$ for $2 \mathrm{~min}$, and chilled on ice. The reverse transcription buffer, dNTPs, RiboLock, and Maxima Reverse Transcriptase (Thermo Scientific) were added in accordance to the manufacturer's instructions, and the mixtures were incubated for $30 \mathrm{~min}$ at $50^{\circ} \mathrm{C}$. The samples were mixed with stop buffer $(8 \mathrm{M}$ urea, $20 \mathrm{mM}$ EDTA, $2 \times \mathrm{TBE}$ ) and analyzed by $20 \%$ PAGE (19:1) together with radiolabeled oligonucleotide markers.

DNA duplex free energy calculation. DNA duplex free energies were analyzed based on nearest-neighbor thermodynamics ${ }^{60,61}$. Briefly, the Python script was written to read a sequence from a text file, calculate the DNA duplex free energy of dinucleotides, sum these values over an 8-base window and report these sums for the first base of the central nucleotide of the window (e.g., the sum for the first window base $1-8$ will be reported for base 4 ).

Reporting summary. Further information on research design is available in the Nature Research Reporting Summary linked to this article.

\section{Data availability}

The data that supports this study are available from the corresponding author upon reasonable request. The cryo-EM density maps have been deposited in EMDataBank under accession codes EMDB: EMD-21879 (RPc), EMD-21880 (RPo), EMD-21881 (RP1-DksA/ppGpp), and EMD-21883 (RP2-DksA/ppGpp). Atomic coordinates for the reported cryo-EM structures have been deposited with the Protein Data Bank under accession numbers 7KHC (RPc), 7KHB (RPo), 7KHI (RP1-DksA/ppGpp), and 7KHE (RP2-DksA/ppGpp). Source data are provided with this paper.

\section{Received: 2 June 2020; Accepted: 14 December 2020;}

Published online: 22 January 2021

\section{References}

1. Nomura, M. Regulation of ribosome biosynthesis in Escherichia coli and Saccharomyces cerevisiae: diversity and common principles. J. Bacteriol. 181, 6857-6864 (1999)

2. Zengel, J. M. \& Lindahl, L. Diverse mechanisms for regulating ribosomal protein synthesis in Escherichia coli. Prog. Nucleic Acid Res. Mol. Biol. 47, 331-370 (1994).

3. Bremer, H. \& Dennis, P. P. Modulation of chemical composition and other parameters of the cell at different exponential growth rates. EcoSal Plus 3, 1-49 (2008).

4. Gourse, R. L. et al. Transcriptional responses to ppGpp and DksA. Annu. Rev. Microbiol. 72, 163-184 (2018).

5. Ross, W. et al. A third recognition element in bacterial promoters: DNA binding by the alpha subunit of RNA polymerase. Science 262, 1407-1413 (1993).

6. Winkelman, J. T. \& Gourse, R. L. Open complex DNA scrunching: a key to transcription start site selection and promoter escape. Bioessays 39, 1600193 (2017)

7. Gaal, T., Bartlett, M. S., Ross, W., Turnbough, C. L. Jr. \& Gourse, R. L. Transcription regulation by initiating NTP concentration: rRNA synthesis in bacteria. Science 278, 2092-2097 (1997).

8. Cashel, M. \& Gallant, J. Two compounds implicated in the function of the RC gene of Escherichia coli. Nature 221, 838-841 (1969). 
9. Potrykus, K. \& Cashel, M. (p)ppGpp: still magical? Annu. Rev. Microbiol. 62, 35-51 (2008)

10. Hauryliuk, V., Atkinson, G. C., Murakami, K. S., Tenson, T. \& Gerdes, K. Recent functional insights into the role of (p)ppGpp in bacterial physiology. Nat. Rev. Microbiol. 13, 298-309 (2015).

11. Molodtsov, V. et al. Allosteric effector ppGpp potentiates the inhibition of transcript initiation by DksA. Mol. Cell 69, 828-839.e5 (2018).

12. Murakami, K. S., Masuda, S., Campbell, E. A., Muzzin, O. \& Darst, S. A. Structural basis of transcription initiation: an RNA polymerase holoenzymeDNA complex. Science 296, 1285-1290 (2002).

13. Zhang, Y. et al. Structural basis of transcription initiation. Science 338, 1076-1080 (2012).

14. Chen, J. et al. Stepwise Promoter Melting by Bacterial RNA Polymerase. Mol. Cell 78, 275-288.e6 (2020).

15. Sreenivasan, R. et al. Fluorescence-detected conformational changes in duplex DNA in open complex formation by Escherichia coli RNA polymerase: upstream wrapping and downstream bending precede clamp opening and insertion of the downstream duplex. Biochemistry 59, 1565-1581 (2020).

16. Ko, J. \& Heyduk, T. Kinetics of promoter escape by bacterial RNA polymerase: effects of promoter contacts and transcription bubble collapse. Biochem J. 463, 135-144 (2014).

17. Karpen, M. E. \& deHaseth, P. L. Base flipping in open complex formation at bacterial promoters. Biomolecules 5, 668-678 (2015).

18. Newlands, J. T., Ross, W., Gosink, K. K. \& Gourse, R. L. Factor-independent activation of Escherichia coli rRNA transcription. II. characterization of complexes of rrnB P1 promoters containing or lacking the upstream activator region with Escherichia coli RNA polymerase. J. Mol. Biol. 220, 569-583 (1991).

19. Murakami, K., Kimura, M., Owens, J. T., Meares, C. F. \& Ishihama, A. The two alpha subunits of Escherichia coli RNA polymerase are asymmetrically arranged and contact different halves of the DNA upstream element. Proc. Natl Acad. Sci. USA 94, 1709-1714 (1997).

20. Lara-Gonzalez, S. et al. The RNA polymerase alpha subunit recognizes the DNA shape of the upstream promoter element. Biochemistry 59, 4523-4532 (2020).

21. Fujita, N., Endo, S. \& Ishihama, A. Structural requirements for the interdomain linker of alpha subunit of Escherichia coli RNA polymerase. Biochemistry 39, 6243-6249 (2000).

22. Sreenivasan, R. et al. Fluorescence resonance energy transfer characterization of DNA wrapping in closed and open Escherichia coli RNA polymeraselambdaP(R) promoter complexes. Biochemistry 55, 2174-2186 (2016).

23. Doniselli, $\mathrm{N}$. et al. New insights into the regulatory mechanisms of ppGpp and DksA on Escherichia coli RNA polymerase-promoter complex. Nucleic Acids Res. 43, 5249-5262 (2015).

24. Feklistov, A. \& Darst, S. A. Structural basis for promoter-10 element recognition by the bacterial RNA polymerase sigma subunit. Cell 147, $1257-1269$ (2011)

25. Murphy, H. \& Cashel, M. Isolation of RNA polymerase suppressors of a (p) ppGpp deficiency. Methods Enzymol. 371, 596-601 (2003).

26. Rutherford, S. T., Villers, C. L., Lee, J. H., Ross, W. \& Gourse, R. L. Allosteric control of Escherichia coli rRNA promoter complexes by DksA. Genes Dev. 23, 236-248 (2009).

27. NandyMazumdar, M. et al. RNA polymerase gate loop guides the nontemplate DNA strand in transcription complexes. Proc. Natl Acad. Sci. USA 113, 14994-14999 (2016).

28. Haugen, S. P., Ross, W., Manrique, M. \& Gourse, R. L. Fine structure of the promoter-sigma region 1.2 interaction. Proc. Natl Acad. Sci. USA 105, 3292-3297 (2008).

29. Winkelman, J. T., Chandrangsu, P., Ross, W. \& Gourse, R. L. Open complex scrunching before nucleotide addition accounts for the unusual transcription start site of E. coli ribosomal RNA promoters. Proc. Natl Acad. Sci. USA 113, E1787-E1795 (2016).

30. Pupov, D., Petushkov, I., Esyunina, D., Murakami, K. S. \& Kulbachinskiy, A. Region 3.2 of the sigma factor controls the stability of rRNA promoter complexes and potentiates their repression by DksA. Nucleic Acids Res. 46, 11477-11487 (2018)

31. Henderson, K. L. et al. Mechanism of transcription initiation and promoter escape by E. coli RNA polymerase. Proc. Natl Acad. Sci. USA 114, E3032-E3040 (2017).

32. Murakami, K. S., Masuda, S. \& Darst, S. A. Structural basis of transcription initiation: RNA polymerase holoenzyme at 4 A resolution. Science 296, 1280-1284 (2002).

33. Mazumder, A. \& Kapanidis, A. N. Recent advances in understanding sigma70dependent transcription initiation mechanisms. J. Mol. Biol. 431, 3947-3959 (2019).

34. Kulbachinskiy, A. \& Mustaev, A. Region 3.2 of the sigma subunit contributes to the binding of the 3 '-initiating nucleotide in the RNA polymerase active center and facilitates promoter clearance during initiation. J. Biol. Chem. 281 , 18273-18276 (2006)

35. Kubori, T. \& Shimamoto, N. A branched pathway in the early stage of transcription by Escherichia coli RNA polymerase. J. Mol. Biol. 256, 449-457 (1996).

36. Stumper, S. K. et al. Delayed inhibition mechanism for secondary channel factor regulation of ribosomal RNA transcription. eLife 8, e40576 n(2019).

37. Parshin, A. et al. DksA regulates RNA polymerase in Escherichia coli through a network of interactions in the secondary channel that includes Sequence Insertion 1. Proc. Natl Acad. Sci. USA 112, E6862-E6871 (2015).

38. Chen, J. et al. E. coli TraR allosterically regulates transcription initiation by altering RNA polymerase conformation. eLife 8, e49375 (2019).

39. Leibman, M. \& Hochschild, A. A sigma-core interaction of the RNA polymerase holoenzyme that enhances promoter escape. $E M B O J . \mathbf{2 6}$, 1579-1590 (2007).

40. Narayanan, A. et al. Cryo-EM structure of Escherichia coli sigma(70) RNAP and promoter DNA complex revealed a role of sigma non-conserved region during the open complex formation. J. Biol. Chem. 293, 7367-7375 (2018).

41. Glyde, R. et al. Structures of bacterial RNA polymerase complexes reveal the mechanism of DNA loading and transcription initiation. Mol. Cell 70, 1111-1120.e3 (2018).

42. Henderson, K. L. et al. RNA polymerase: step-by-step kinetics and mechanism of transcription initiation. Biochemistry 58, 2339-2352 (2019).

43. Sanchez-Vazquez, P., Dewey, C. N., Kitten, N., Ross, W. \& Gourse, R. L. Genome-wide effects on Escherichia coli transcription from ppGpp binding to its two sites on RNA polymerase. Proc. Natl Acad. Sci. USA 116, 8310-8319 (2019).

44. Gummesson, B., Lovmar, M. \& Nystrom, T. A proximal promoter element required for positive transcriptional control by guanosine tetraphosphate and DksA protein during the stringent response. J. Biol. Chem. 288, 21055-21064 (2013).

45. Paul, B. J., Berkmen, M. B. \& Gourse, R. L. DksA potentiates direct activation of amino acid promoters by ppGpp. Proc. Natl Acad. Sci. USA 102, 7823-7828 (2005).

46. Girard, M. E. et al. DksA and ppGpp regulate the sigma(S) stress response by activating promoters for the small RNA DsrA and the anti-adapter protein IraP. J. Bacteriol. 200, 1-12 (2018).

47. Costanzo, A. et al. ppGpp and DksA likely regulate the activity of the extracytoplasmic stress factor sigmaE in Escherichia coli by both direct and indirect mechanisms. Mol. Microbiol. 67, 619-632 (2008).

48. Li, L., Fang, C., Zhuang, N., Wang, T. \& Zhang, Y. Structural basis for transcription initiation by bacterial ECF sigma factors. Nat. Commun. 10, 1153 (2019)

49. $\mathrm{Xu}$, J. et al. Crl activates transcription by stabilizing active conformation of the master stress transcription initiation factor. eLife 8, e50928 (2019).

50. Murakami, K. S. X-ray crystal structure of Escherichia coli RNA polymerase sigma70 holoenzyme. J. Biol. Chem. 288, 9126-9134 (2013)

51. Zivanov, J. et al. New tools for automated high-resolution cryo-EM structure determination in RELION-3. elife 7, e42166 (2018)

52. Zheng, S. Q. et al. MotionCor2: anisotropic correction of beam-induced motion for improved cryo-electron microscopy. Nat. Methods 14, 331-332 (2017).

53. Zhang, K. Gctf: real-time CTF determination and correction. J. Struct. Biol. 193, 1-12 (2016).

54. Punjani, A., Rubinstein, J. L., Fleet, D. J. \& Brubaker, M. A. cryoSPARC: algorithms for rapid unsupervised cryo-EM structure determination. Nat. Methods 14, 290-296 (2017).

55. Pettersen, E. F. et al. UCSF Chimera-a visualization system for exploratory research and analysis. J. Comput Chem. 25, 1605-1612 (2004).

56. Afonine, P. V. et al. Joint X-ray and neutron refinement with phenix.refine. Acta Crystallogr. D. Biol. Crystallogr. 66, 1153-1163 (2010).

57. Emsley, P. \& Cowtan, K. Coot: model-building tools for molecular graphics. Acta Crystallogr. D. Biol. Crystallogr. 60, 2126-2132 (2004).

58. Pettersen, E. F. et al. UCSF ChimeraX: structure visualization for researchers, educators, and developers. Protein Sci. 30, 70-82 (2021).

59. Svetlov, V. \& Artsimovitch, I. Purification of bacterial RNA polymerase: tools and protocols. Methods Mol. Biol. 1276, 13-29 (2015).

60. SantaLucia, J. Jr. A unified view of polymer, dumbbell, and oligonucleotide DNA nearest-neighbor thermodynamics. Proc. Natl Acad. Sci. USA 95 1460-1465 (1998).

61. Dienemann, C., Schwalb, B., Schilbach, S. \& Cramer, P. Promoter distortion and opening in the RNA polymerase II cleft. Mol. Cell 73, 97-106.e4 (2019).

\section{Acknowledgements}

We thank Carol Bator, Mike Carnegie, and Jean-Paul Armache at Penn State for supporting the cryo-EM data collection and processing. We thank Christian Dienemann at 
the Max Planck Institute for providing the DNA duplex free energy calculation methodology. We thank Yu Zhang at the Shanghai Institute of Plant Physiology and Ecology and Yu Feng at Zhejiang University School of Medicine for providing technical adovice. This research was, in part, supported by the National Cancer Institute's National CryoEM Facility at the Frederick National Laboratory for Cancer Research under contract HSSN261200800001E. This work was supported by NIH grants (R01 GM087350 and R35 GM131860 to K.S.M.) and Russian Science Foundation (grant 19-14-00359 to D.E.) and the Russian Foundation for Basic Research (grant 18-34-20095 to A.K.).

\section{Author contributions}

Y.S. prepared samples and cryo-EM grids. Y.S. and M.Z.Q. collected and processed cryoEM data. K.S.M. built, refined, and validated the structures. D.P. and D.E. performed biochemical assays under the supervision of A.K. K.S.M. designed and supervised research. All authors participated in the interpretation of the results and in writing the paper.

\section{Competing interests}

The authors declare no competing interests.

\section{Additional information}

Supplementary information is available for this paper at https://doi.org/10.1038/s41467020-20776-y.

Correspondence and requests for materials should be addressed to K.S.M.
Peer review information Nature Communications thanks M. Thomas Record Jr., Yu Zhang and the other, anonymous, reviewer(s) for their contribution to the peer review of this work. Peer reviewer reports are available.

Reprints and permission information is available at http://www.nature.com/reprints

Publisher's note Springer Nature remains neutral with regard to jurisdictional claims in published maps and institutional affiliations.

(c) Open Access This article is licensed under a Creative Commons Attribution 4.0 International License, which permits use, sharing, adaptation, distribution and reproduction in any medium or format, as long as you give appropriate credit to the original author(s) and the source, provide a link to the Creative Commons license, and indicate if changes were made. The images or other third party material in this article are included in the article's Creative Commons license, unless indicated otherwise in a credit line to the material. If material is not included in the article's Creative Commons license and your intended use is not permitted by statutory regulation or exceeds the permitted use, you will need to obtain permission directly from the copyright holder. To view a copy of this license, visit http://creativecommons.org/ licenses/by/4.0/.

(C) The Author(s) 2021 